\title{
GESTÃO DE ENERGIA E INDÚSTRIA 4.0*
}

Luciano Lellis Miranda ${ }^{1}$ Ricardo Giacomin ${ }^{2}$

Thiago Turchetti Maia ${ }^{3}$

\section{Resumo}

Este trabalho apresenta os principais conceitos de Indústria 4.0 e discute os benefícios do emprego de alguns de seus mecanismos na construção de um sistema integrado de gestão de energia, em especial a utilização das tecnologias de big data (ou megadados, em português). A tese defendida neste trabalho - a possibilidade de se produzir grande valor a partir da abundância de dados de medição de consumo, de produção e de estados dos equipamentos, tipicamente encontrada em ambientes industriais - é demonstrada através do caso concreto da empresa Aperam South America, em sua planta de Timóteo, MG. Os resultados apresentados neste trabalho ilustram, qualitativamente, os potenciais retornos apurados no desenvolvimento de um sistema com as características descritas.

Palavras-chave: Indústria 4.0; Gestão de energia; Megadados; Inteligência analítica.

\section{ENERGY MANAGEMENT AND INDUSTRY 4.0}

\begin{abstract}
This paper presents the basic concepts of Industry 4.0 and points out the benefits of using its core mechanisms, particularly the big data frameworks, to build an integrated energy management system. The thesis presented here - the opportunity to create value based on the availability of huge amounts of measurement data, material production data and equipment status information, tipically found in industrial IT and automation systems landscapes - is evidenced by a real business case at Aperam South America, in its integrated plant at Timóteo, MG. The results presented in this work illustrate, qualitatively, the potencial return on the investiment on a system based on the aforementioned big data approach.
\end{abstract}

Keywords: Industry 4.0; Energy management; Big data; Predictive analytics.

1 Engenheiro Metalurgista e Meste em Engenharia Mecânica, Assistente Técnico de Processo, Gerência de Excelência Operacional, Aperam South America, Timóteo, MG, Brasil.

2 Bacharel e Mestre em Ciência da Computação, Diretor Executivo, Viridis Soluções em Energia S.A., Belo Horizonte, MG, Brasil.

3 Bacharel em Ciência da Computação, Mestre e Doutor em Engenharia Elétrica, Presidente, Viridis Soluções em Energia S.A., Belo Horizonte, MG, Brasil. 


\section{INTRODUÇÃO}

\subsection{Objetivos}

Este trabalho pretende discutir os principais conceitos de Indústria 4.0 e a sua relação com a disciplina de gestão de energia em grandes consumidores industriais. Serão destacados os maiores desafios relacionados à captura e tratamento de grandes volumes de dados, além do seu devido processamento para um controle e gestão de energia mais efetivos, melhoria de eficiência energética e consequente produção de valor para as empresas. Para ilustração, será apresentado o caso real de emprego de algumas tecnologias recentes na implementação de um Sistema Integrado de Gestão de Energia (SIGE), na Aperam South America, em Timóteo/MG.

\subsection{Indústria 4.0}

Tecnologias emergentes e poderosas têm habilitado o desenvolvimento de novas soluções para a indústria, promovendo uma verdadeira revolução na forma de se planejar, executar e gerenciar a operação das fábricas. Figuram nesse cardápio de novas tecnologias: Internet das Coisas Industriais (IIoT - Industrial Internet of Things, em inglês), megadados (big data, em inglês), impressão 3D, computação em nuvem, redes sem fio de sensores, inteligência analítica, entre outras. Este novo estágio de desenvolvimento, chamado Indústria 4.0, em alusão a uma quarta revolução industrial (veja figura abaixo), tem potencial para redefinir a cadeia de valor e processos na indústria de manufatura, permitindo que células isoladas e otimizadas constituam fluxos de produção completamente integrados, automatizados e otimizados, promovendo maior eficiência e mudando as relações tradicionais entre fornecedores, fábricas e clientes.

18

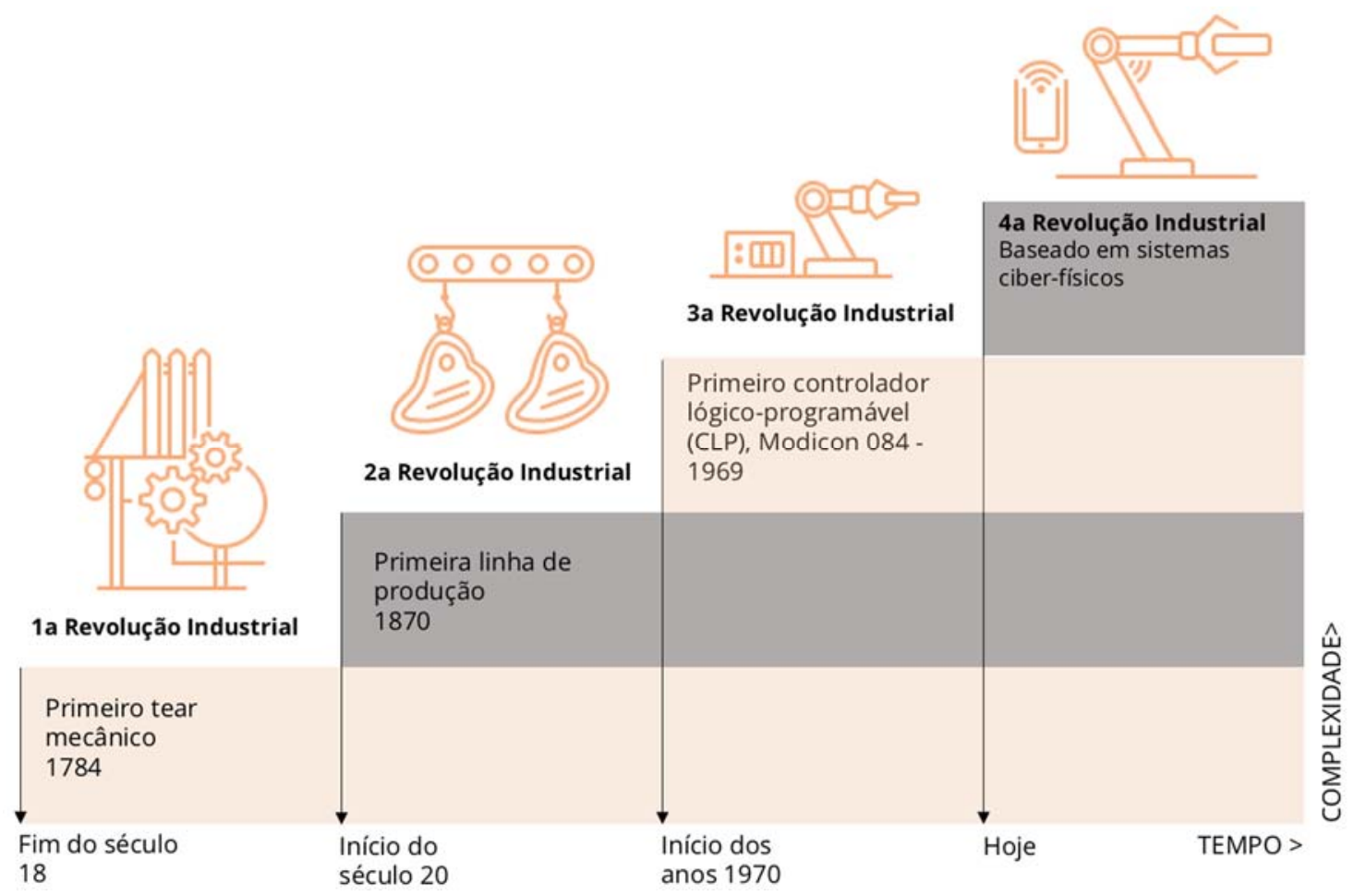

Figura 1. Indústria 4.0. 


\subsection{Megadados}

No repertório tecnológico da Indústria 4.0, ocupa posição de destaque o chamado big data (ou megadados, em português). Em tecnologia da informação, o termo big data se refere ao processo de examinar grandes conjuntos de dados, em formas e tipos variados, com o objetivo de descobrir padrões, correlações, tendências, ou seja, informação útil e de valor. Quando aplicado ao contexto industrial, a abordagem de big data se refere a: 1) a capacidade de rápida digestão de dados, provenientes de inúmeros sensores, sistemas de automação (CLPs, SCADAs, historiadores), sistemas de informação, serviços web, entre outros; 2) o cruzamento ou correlação das principais variáveis com os dados contexto dos equipamentos na fábrica; 3) o processamento e análise estatística dos dados para identificação de ineficiências e oportunidades de melhoria de desempenho, em tempo real.
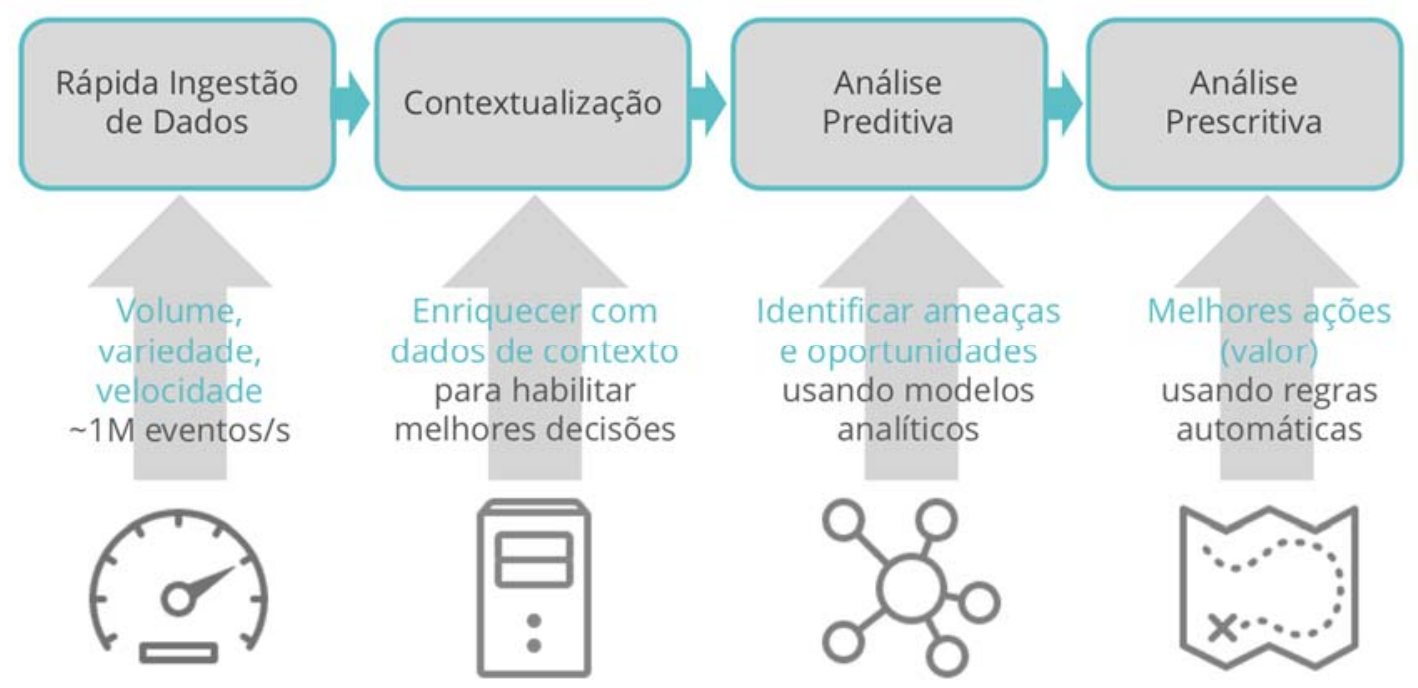

Figura 2. Big data.

\subsection{Os 'Vs' do Big Data: Volume, Velocidade, Variedade, Veracidade e Valor}

Para tornar o conceito de big data mais claro, é comum a tradução de seus principais aspectos em 5 dimensões - volume, velocidade e variedade, veracidade e valor - os chamados 5 Vs do Big Data.

- Volume. As empresas devem ser capazes de coletar, armazenar e processar grandes quantidades de dados, de múltiplas fontes, incluindo transações comerciais, redes sociais e informações de sensores ou dados transmitidos de máquina a máquina.

- Velocidade. Os dados fluem em uma velocidade sem precedentes e devem ser tratados em tempo hábil, tipicamente em tempo real (ou quase real).

- Variedade. Os dados são gerados em todos os tipos e formatos - como dados estruturados, séries temporais, dados numéricos em sistemas de bancos de dados tradicionais, até documentos de texto não estruturados.

- Veracidade. É necessário que sejam estabelecidos processos que garantam o máximo possível a consistência dos dados, para que seu emprego em análises e tomada de decisão produza real valor.

- Valor. Os recursos de inteligência analítica devem permitir a transformação dos grandes volumes de dados em algo útil para o negócio, vetor de melhoria de desempenho. 


\subsection{Big Data e Gestão de Energia}

Tipicamente, sistemas de gestão de energia lidam com os principais indicadores de desempenho energético (IDEs) em uma resolução mensal e em visões consolidadas por linha, área, ou até mesmo visões globais, para toda uma planta. Nesse cenário de falta de visão de informações de desempenho em tempo real, há pouco espaço para a reação de operadores e responsáveis pelos processos, nos momentos em que as ineficiências ocorrem. Não é possível responder a perguntas básicas como: "há algum vazamento na tubulação de gás?", "o consumo de energia está de acordo com o padrão, considerando o material sendo produzido?", "considerando todas as restrições técnicas e de contrato, como devemos atender à demanda energética para o plano de produção de hoje?". O resultado deste "voo cego" é, naturalmente, baixo desempenho.

Neste contexto, são necessários sistemas sofisticados de software capazes de, em tempo real, capturar e validar grandes volumes de dados de consumo e geração de energia, analisá-los e reconhecer padrões, correlacionar variáveis e detectar ineficiências energéticas. Um sistema com essas capacidades, empregando a abordagem de big data para endereçar os avançados desafios de gestão de energia, é um grande aliado na busca pelo custo ideal e excelência operacional.

Um sistema de gestão de energia avançado, aplicado em contexto industrial, deve ser capaz de se integrar e ler grande volume de dados, incluindo (mas não se limitando a): dados da infraestrutura de medição, diretamente ou através de concentradores (como historiadores); informações de produção realizada em cada equipamento, a partir dos sistemas de automação ou de gestão da produção (como MES Manufacturing Execution Systems e ERP - Enterprise Resource Planning); dados do estado de cada equipamento, provenientes de sistemas de supervisão e controle; e dados do ambiente operacional, como, por exemplo, de temperatura ambiente. A velocidade de leitura dessas informações deve ser próxima do tempo real (amostragem mínima de uma leitura por minuto), assim como o seu processamento, permitindo a pronta detecção e reação a ocorrências de ineficiência. Igualmente importante é a capacidade de lidar com a variedade de formatos de dados, incluindo séries temporais, dados transacionais (ou relacionais) e dados não estruturados. Por fim, é imperativo que tal sistema seja capaz de avaliar a veracidade e qualidade dos dados digeridos, detectar problemas e higienizá-los à medida que ocorram.

A disciplina de controle fino, preciso e continuado garante maior eficiência e captura valor para a empresa. Esta tese foi validada pelo projeto de implantação de um software de gestão de energia na Aperam, conforme relatado nas seções seguintes deste trabalho.

\section{MATERIAIS E MÉTODOS}

\subsection{Sistema Integrado de Gestão de Energia}

Com o intuito de melhorar o seu desempenho energético, a Aperam South America desenvolveu uma gestão sistemática e ágil das informações relacionadas ao consumo, geração, aplicação, suprimento e custos energéticos. $O$ projeto desenvolvido, denominado SIGE (Sistema Integrado de Gestão de Energia), incluiu a implantação e configuração de um software integrado, o Viridis Energy Resources Optimizer, da empresa Viridis Soluções em Energia, construído sobre os pilares tecnológicos da Indústria 4.0, especialmente a tecnologia de big data. 
O escopo de utilização deste software foi definido por: todas as áreas produtivas da usina integrada da Aperam, em Timóteo/MG; todos os insumos energéticos, primários e secundários; diversos públicos internos, incluindo a própria área de energia e utilidades, as áreas produtivas, a controladoria, os gestores de contratos, analistas de instrumentação, entre outros.

\subsection{Organização}

A figura abaixo apresenta a organização do sistema desenvolvido.

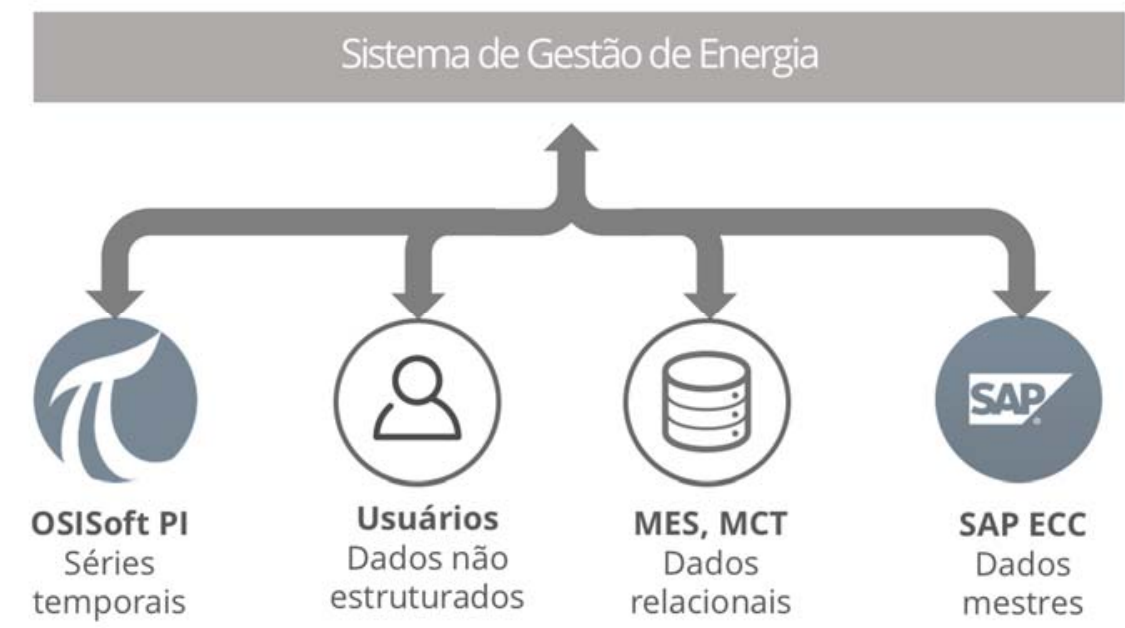

Figura 3. Organização do sistema de gestão de energia.

Como descrito acima, as principais fontes de dados para o sistema de energia são:

- Sistema PIMS da OsiSoft, com os dados de séries temporais, principalmente relacionados ao consumo e à geração de energia.

- Usuários, que informam dados de medição (para os medidores não conectados à rede) e dados não estruturados, como anotações vinculadas às séries.

- Sistema MES, com informações das ordens de produção executadas em cada equipamento, incluindo dados de aço, grupo, família, largura, espessura, etc.

- Sistema de controle de paradas, a partir do qual são apuradas as informações dos estados dos equipamentos (como "desligado", "disponível", "produzindo", "conservando temperatura", etc.)

- Sistema SAP, para leitura de dados mestres como locais de instalação e centros de custo.

- Outros sistemas, como sistemas de balanças rodoviárias, para leitura de entradas e saídas de insumos energéticos, para fechamento do balanço energético das unidades de armazenamento.

\subsection{Volume, Velocidade e Veracidade}

O sistema foi configurado para capturar e armazenar os dados de mais de 2000 medidores, necessários à plena gestão de 15 insumos energéticos (incluindo energia elétrica, gases do ar, gás natural, combustíveis, gás siderúrgico e águas) em mais de 1000 equipamentos. A uma taxa de uma leitura por minuto, todos os medidores são utilizados para o cálculo de aproximadamente 1800 indicadores de desempenho 
energético (IDEs). Com essa configuração, um total de aproximadamente 6 bilhões de registros por ano são armazenados e processados.

Para garantir a qualidade dos dados utilizados nas análises, foi configurada uma heurística de higienização das medições para cada problema possível em cada medidor cadastrado. Por exemplo, foi definido para cada medidor quais os limites operacionais (limite superior e inferior) e a correção que o sistema deve fazer em caso de pontos fora dessa faixa. Exemplos de heurísticas de higienização incluem: média dos últimos pontos, tendência, constante, medição alternativa (caso existente), entre outras.

\subsection{Inteligência Analítica e Geração de Valor}

Uma série de funções analíticas do sistema de gestão de energia foram ativadas para permitir a identificação de problemas na operação e, por conseguinte, influenciar positivamente no desempenho energético.

A interação dos operadores e engenheiros com essas funções se dá de diversas formas: 1) através de relatórios programados para regularmente reportarem situações de interesse, como a tradução da performance em ganhos ou perdas financeiras; 2) através de painéis de controle, definidos e configurados pelos próprios usuários, para atender às suas necessidades de controle em tempo real, consolidando indicadores e variáveis de contexto; 3 ) e, principalmente, através de alertas em tempo real e para o público correto, com a indicação de situações que requerem investigação e intervenção.

Por exemplo, os dados de produção provenientes do sistema MES são utilizados como variáveis analíticas para os indicadores de consumo e geração. O objetivo é monitorar padrões de consumo específico, estratificados por variáveis como aço, família ou geometria dos produtos. Alertas foram configurados para notificador operadores, em tempo real, de desvios em relação ao modelo ideal (meta) de consumo.

A figura abaixo apresenta uma interface de usuário do sistema implantado, com destaque para o cálculo e monitoramento das metas de consumo absoluto em função das características da produção realizada, consideradas dimensões analíticas dos indicadores de desempenho energético.

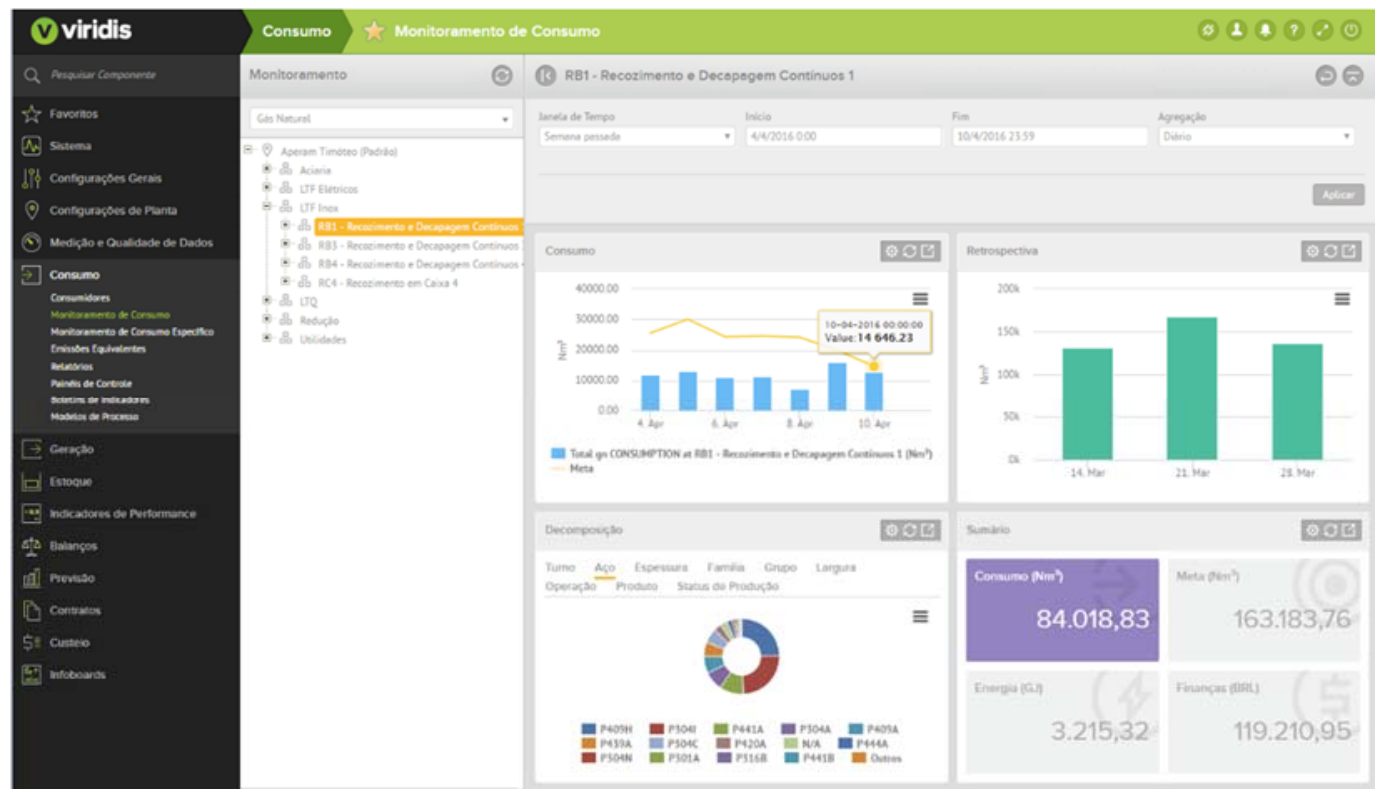

Figura 4. Exemplo de interface de monitoramento de consumo específico e absoluto. 
Para ilustrar a potencial geração de valor, são apresentados dois casos concretos e muito frequentes, intimamente relacionados à degradação da performance energética, que apenas um sistema informatizado permite à Aperam endereçar: controle de energia inútil e detecção de vazamentos.

As entradas para a análise do primeiro caso - gestão de energia inútil - são o estado e o contexto de cada equipamento da planta e o indicador de consumo absoluto calculado. As informações de estado e de produção/contexto do equipamento são lidas de um sistema de MES. A meta de consumo é definida em função dessas variáveis. Por exemplo, equipamentos parados não devem consumir nada. Equipamentos produzindo devem consumir de acordo com o que está sendo produzido (quantidade e produto). Alguns equipamentos, como fornos, podem estar conservando temperatura e, neste estado, devem apresentar um consumo mínimo. Ao confrontar o indicador de consumo com o padrão esperado para cada estado, o sistema pode, em tempo real, identificar e alertar sobre energia empregada sem finalidade, ou acima dos padrões esperados.

A detecção de vazamentos nas redes de energia, principalmente gases, é feita através da análise de balanceamento dos medidores da rede. Um algoritmo de reconciliação de dados de medição, que também considera as imprecisões dos medidores e o leiaute da rede, identifica circuitos com frequente desbalanceamento das medições e, assim, sugere possíveis pontos de vazamento. Esta análise, computacionalmente cara, não poderia ser realizada continuamente por operadores ou engenheiros, sem o suporte de um sistema informatizado.

\section{RESULTADOS E DISCUSSÃO}

A implantação do sistema integrado de gestão de energia, baseado nas técnicas de big data, trouxe resultados relevantes para a Aperam South America. Servindo como uma plataforma de integração de dados, pessoas, métodos e processos, o software tem sido usado como importante ferramenta de análise, controle e gestão da eficiência energética pela empresa, melhorando seus principais indicadores. A agora possível capacidade de se processar grandes volumes de dados e correlacionar com informações de contexto dos equipamentos, permite que metas de eficiência sejam justamente definidas e sua execução monitorada em tempo real.

A abordagem discutida neste trabalho permitiu que ganhos como os apresentados abaixo, de forma qualitativa, fossem realizados. Tais ganhos são provenientes de uma série de medidas tomadas pela operação, cotidiana e sistematicamente, a partir das análises e alertas do software, em cada um dos processos de produção.

- Detecção de vazamento na rede interna de distribuição de argônio, evitando custos elevados e desnecessários. Sem um sistema capaz de capturar todas as medições em tempo real e realizar o balanceamento físico da rede de medidores, através de um procedimento de reconciliação de dados, tal detecção aconteceria provavelmente após muito desperdício.

- Detecção de uso indevido e ineficiente (economicamente) de insumos energéticos alternativos, em equipamentos que trabalham com mais de um insumo para a mesma finalidade.

- Detecção de metas mal calibradas, através da manutenção automática do modelo de consumo de cada insumo por cada equipamento, do monitoramento do histórico e melhores desempenhos, e do confronto com as metas estabelecidas pela empresa. 
- Detecção automática de "energia inútil", decorrente do consumo elevado (ou incoerente) em períodos em que os equipamentos permanecem desligados, disponíveis ou conservando temperatura, ou seja, em períodos de nãoprodução.

- Rápida detecção de falhas de medição, através da devida configuração dos recursos do software para a identificação de problemas de qualidade de dados e a higienização dos mesmos.

\section{CONCLUSÃO}

Este estudo apresenta uma visão geral do conceito e tecnologias relacionadas à chamada Indústria 4.0, e destaca o emprego de uma arquitetura de big data para a construção de um sistema de gestão de energia. Pela coleta de dados em grande volume e em diversos formatos, e pela construção de recursos de inteligência analítica para processamento e tradução dos dados em valor, um sistema integrado de gestão de energia pode ser uma importante ferramenta para as empresas vencerem o desafio de melhorar sua eficiência energética e, de maneira mais ampla, operacional. A tese defendida neste trabalho é a de adoção de um controle e gestão de energia em tempo real, feita de forma sistemática e contínua. A integração dos indicadores de energia nas práticas de gestão de produção é fator fundamental para a melhoria de desempenho energético.

\section{REFERÊNCIAS}

1 Shrouf F., Ordieres J., Miragliotta G. Smart factories in Industry 4.0: A review of the concept and of energy management approached in production based on the Internet of Things paradigm. 2014 IEEE International Conference on Industrial Engineering and Engineering Management. 2014; Conference Publications: 697-701.

2 Han, J; Kamber, M. Data Mining: Concepts and Techniques. 2nd Edition. San Francisco: Elsevier, 2006.

3 Hong Kong Electronic Industries Association (HKEIA). Guidebook for ISO 50001: Energy Management System. Hong Kong, Mach 2013.

4 Miranda, L. L., Martins, Machado, G. Sistema Integrado de Gestão de Energia em uma Empresa Siderúrgica. Trabalho de Conclusão do Curso de Pós-Graduação em Eficiência Energética Aplicada aos Processos Produtivos. UFSM, Santa Maria, RS. 